\title{
Th1 and Th2 mediate acute graft-versus-host disease, each with distinct end-organ targets
}

\author{
Boris Nikolic, ${ }^{1}$ Seika Lee, ${ }^{1}$ Roderick T. Bronson, ${ }^{2}$ Michael J. Grusby, ${ }^{3}$ and Megan Sykes ${ }^{1}$ \\ ${ }^{1}$ Bone Marrow Transplantation Section, Transplantation Biology Research Center, Surgical Service, \\ Massachusetts General Hospital/Harvard Medical School, Boston, Massachusetts, USA \\ ${ }^{2}$ US Department of Agriculture Human Nutrition Research Center on Aging at Tufts University, Boston, Massachusetts, USA \\ ${ }^{3}$ Department of Immunology and Infectious Diseases, Harvard School of Public Health and Department of Medicine \\ at Harvard Medical School, Boston, Massachusetts, USA \\ Address correspondence to: Megan Sykes, Bone Marrow Transplantation Section, Transplantation Biology Research Center, \\ Massachusetts General Hospital, MGH East, Building 149-5102, 13th Street, Boston, Massachusetts 02129, USA. \\ Phone: (617) 726-4070; Fax: (617) 724-9892; E-mail: megan.sykes@tbrc.mgh.harvard.edu.
}

Received for publication July 16, 1999, and accepted in revised form March 6, 2000.

STAT4 and STAT6 are transcription factors that play crucial roles in responding to IL-12 and IL-4, respectively. STAT4 gene knockout $\left(S T A T 4^{-/}\right)$mice have markedly reduced Th1 responses and enhanced Th2 responses. STAT6 ${ }^{-/-}$mice show the inverse phenotype. We compared the ability of bone marrow transplantation (BMT) with the inclusion of spleen cells from STAT6 ${ }^{-{ }^{-}}, \mathrm{STAT4}^{-{ }^{-}}$, and wildtype (WT) mice to produce graft-versus-host disease (GVHD) in lethally irradiated MHC-mismatched recipients. Acute GVHD mortality was more rapid when induced by cells from $S T A T 6^{-/-}$mice than when induced by STAT4 ${ }^{-/-}$cells. However, cells from STAT4 ${ }^{-/-}$and STAT6 ${ }^{-/-}$donors both induced delayed GVHD mortality compared with WT controls, or compared with combined STAT4 ${ }^{-/-}$and $S T A T 6^{-/}$cells, indicating a contribution of both Th1 cells and Th2 cells to acute GVHD. Recipients of $S T A T 6^{-/}$BMT showed evidence of acute GVHD with severe diarrhea and marked weight loss. Recipients of $S T A T 4^{-/}$BMT showed signs of GVHD with only initial transient weight loss and later development of severe skin GVHD. Histopathology showed that Th2 responses were required for the induction of both hepatic and severe skin GVHD. In contrast, both Th1 cells and Th2 cells were capable of causing intestinal pathology of GVHD. Our studies demonstrate an additive role for Th1 and Th2 cells in producing acute GVHD, and suggest a cytokine-directed approach to treating end-organ manifestations of GVHD.

J. Clin. Invest. 105:1289-1298 (2000).

\section{Introduction}

Despite major advances, the full therapeutic potential of bone marrow transplantation (BMT) has not yet been achieved. Graft-versus-host disease (GVHD), arising from an attack of donor $T$ cells against alloantigens of the recipient, is a major complication of allogeneic BMT, and prohibits the successful application of BMT across extensive human leukocyte antigen barriers. Despite the morbidity and death it causes, GVHD has been associated with 2 clinical benefits. Donor T cells exert graft-versus-leukemia effects by destroying residual leukemic cells, and also enhance the engraftment process, probably by destroying residual host $T$ cells that survive intensive conditioning regimens (1). Therefore, separation of the beneficial engraftment-promoting and anti-leukemic effects of donor T cells from their ability to induce GVHD is a major goal of research in BMT.

Two distinct functional cytokine secretion patterns, termed Th1 and Th2, have been defined for murine as well as human $\mathrm{T}$ helper cells. The Th1 response is characterized by the production of IFN- $\gamma$, IL-2, and TNF- $\beta$, by the stimulation of delayed-type hypersensitivity reactions, and by the production of high-affin- ity Fc $\gamma$ receptor-binding Abs ( $\operatorname{IgG}_{2 a}$ in the mouse) (2). Characteristic of the Th2 response in the mouse is the production of IL-4 and IL-5, and the stimulation of the production of $\mathrm{IgE}$ and $\mathrm{IgG}_{1}$ Abs (2). These cell types are cross-regulatory in vitro, and the balance of these cells in vivo determines the character of cellmediated immune and inflammatory responses (2). Recently, it has been suggested that the Th1/Th2 polarization of $\mathrm{T}$ helper cell subsets plays an important role in the development of GVHD. In both mice and humans, a "cytokine storm" related to the Th1 phenotype has been found to correlate with the development of GVHD $(3,4)$. Studies have shown that in vivo injection of IL-2 plus IL-4 into donor mice resulted in the generation of $\mathrm{CD}^{+} \mathrm{IL}^{-4}-$ and IL-10-producing Th2 cells that, upon transfer into nonirradiated $\mathrm{F}_{1}$ or sublethally irradiated mice, inhibited GVHD lethality $(5,6)$. Furthermore, production of Th2 cytokines induced by G-CSF administration has been associated with inhibitory effects on the development of GVHD (7). In another study, transplantation of in vitro-polarized murine Th2 cells resulted in a significant increase of survival after BMT across minor histocompatibility antigenic barriers (8). 
However, some other studies of the therapeutic approach of using a Th1 to Th2 switch for the prevention or treatment of GVHD have suggested that the roles of Th 1 and Th 2 cells in GVHD may be far more complicated than was previously believed. Altering the development of GVHD or treating ongoing GVHD by direct administration of Th2 cytokines in vivo has not been successful. In 1 study, recipients of IL-4 showed increased mortality and equivalent, histopathological changes in the skin and liver compared with mice receiving no cytokine. It has also been shown that the exogenous administration of IL-10 was not toxic per se, but did accelerate GVHD lethality $(9,10)$. Furthermore, recent studies using $I F N-\gamma$ or $I L-4$ gene knockout mice as donors showed that the absence of IFN- $\gamma$ is still associated with severe acute GVHD $(11,12)$, whereas the inability of donors to produce IL-4 can be associated with less severe GVHD (12).

To elucidate the contribution of T helper cell subsets, we initiated a study using gene knockout mice that were capable of mounting only Th1 or Th2 responses as donors. Interactions between cytokines and their receptors leads to the activation of multiple signaling molecules, including the family of signal transducer and activator of transcription (STAT) proteins. STAT4 is a member of this family that is essential for responsiveness to IL-12. Mice with a disrupted STAT4 gene (STAT4-/mice) have impaired IL-12 responsiveness of NK and T cells, lack Th1 responsiveness, and have enhanced Th2 cell function $(13,14)$. STAT6 is activated in response to IL-4 and IL-13. Therefore, Tlymphocytes from STAT6 ${ }^{-/-}$ mice have impaired IL-4 and IL-13 responsiveness, and almost completely lack Th2 responses, but show enhanced Th1 responses (15-17). We assessed the role of Th1 cells and Th2 cells in GVHD by transferring bone marrow cells (BMC) and spleen cells from STAT4 ${ }^{--}$and $S T A T 6^{-/}$mice to lethally irradiated, fully MHC-mismatched BMT recipients. Our results demonstrated that both Th1 cells and Th 2 cells contribute to the development and severity of acute GVHD.

\section{Methods}

Mice. Specific pathogen-free female C57BL/6 (B6, H-2 ${ }^{\mathrm{b}}$, $\left.\mathrm{K}^{\mathrm{b}} \mathrm{I}^{\mathrm{b}} \mathrm{D}^{\mathrm{b}}\right)$ and $\mathrm{BALB} / \mathrm{c}\left(\mathrm{H}-2^{\mathrm{d}}, \mathrm{K}^{\mathrm{d}} \mathrm{I}^{\mathrm{d}} \mathrm{D}^{\mathrm{d}}\right)$ mice were purchased from the Frederick Cancer Research Facility (NCI Laboratories, Frederick, Maryland, USA). STAT4 and STAT6 gene knockout mice were generated on the $129 \times$ $\mathrm{BALB} / \mathrm{c}$ background in M. Grusby's laboratory $(13,16)$, and were backcrossed to the BALB/c background 5-10 times. All donor lines were $\mathrm{H}-2^{\mathrm{d}}$ homozygotes. Mice were housed in sterilized microisolator cages and received autoclaved feed and autoclaved, acidified drinking water. Preparation of BMC, spleen cells, and T-cell depleted BMC. $\mathrm{BMC}$ and splenocyte suspensions were prepared as described (18). Host-type (B6) BMC were depleted of T cells with mAbs against CD4 (GK1.5 in ascites, 1:1,000 dilution) (19) and CD8 (2.43 in ascites, 1:1,500 dilution) (20), plus low-toxicity rabbit complement (1:16 dilution), as described previously (21).
Total body irradiation and BMT. Recipient mice were lethally irradiated $\left(9.75 \mathrm{~Gy},{ }^{137} \mathrm{Cs}\right.$ source) and were reconstituted within $4-8$ hours with a single $(1 \mathrm{~mL})$ intravenous inoculum. For each experiment we compared the number of $\mathrm{CD}^{+}$and $\mathrm{CD} 8^{+} \mathrm{T}$ cells in the spleens of wild-type (WT), STAT4-/-, and STAT6 ${ }^{-{ }^{-}}$ mice, and we did not observe significant differences in the percentages of each cell type. To avoid bias from cage-related effects, animals were randomized as described (22).

Flow cytometry and $m A b$ s. Spleen cells were analyzed by 2-color flow cytometry on a FACScan (Becton Dickinson, Mountain View, California, USA). To block nonspecific Fc $\gamma$ receptor binding of labeled Abs, 2.4G2 (rat anti-mouse Fc $\gamma$ receptor $\mathrm{mAb}$ ) (23) was added to the first incubation. To determine the percentage of donorand host-type $\mathrm{T}$ cells in spleen, cells were stained with FITC-conjugated rat anti-mouse CD4 mAb, rat antimouse CD8 mAb (PharMingen, San Diego, California, USA), and biotinylated anti-H2 ${ }^{d}$ mAb 34-2-12 (24). The percentage of donor- and host-type $\mathrm{T}$ cells was determined by subtracting the percentage of HOPC1staining cells from the percentage of $\mathrm{CD}^{+}$and $\mathrm{CD}^{+}$ cells in the same quadrant, as described (11).

Mixed lymphocyte culture (MLC) supernatants. Spleen cells from randomly chosen GVHD control, STAT4-/-, and $S T A T 6^{-/-}$transplant recipients, and from normal $\mathrm{B} 6$ and normal BALB/c mice were resuspended to a concentration of $10^{6}$ cells $/ \mathrm{mL}$ as responders. Stimulators were prepared from normal B6 or BALB/c mice, irradiated with $30 \mathrm{~Gy}$, and resuspended to a concentration of $4 \times 10^{6}$ cells $/ \mathrm{mL}$. Responder and stimulator cell suspensions of $100 \mu \mathrm{L}$ each were plated in 96-well U-bottom tissue culture plates. After incubation for 24 hours, culture supernatants were collected for cytokine analyses, and were immediately aliquoted and frozen.

ELISA for cytokine levels in MLC supernatants and for immunoglobulin levels in sera. Cytokines in MLC supernatants were measured by ELISA using mouse cytokine-specific rat mAbs (IFN- $\gamma$ capture mAb R46A2, IFN- $\gamma$ detecting mAb XMG 1.2; IL-2 capture mAb JES6-1A12, IL-2 detecting mAb JES6-5H4; IL-4 capture mAb R4-6A2BVD4-1D11, IL-4 detecting mAb BVD624G2; IL-10 capture mAb JES5-2A5, IL-10 detecting $\mathrm{mAb}$ SXC-1), and were compared with known concentrations of mouse recombinant cytokines (PharMingen) as described (25). IgE, IgG 1 , and total IgG levels in sera were measured by ELISA using purified mouse immunoglobulin standards and mouse immunoglobulin-specific Abs (IgE capture mAb R35-72, IgE detecting $m A b$ R35-92; IgG 1 capture $\mathrm{mAb}_{\mathrm{A}} \mathrm{A5}-3$, IgG $\mathrm{I}_{1}$ detecting $\mathrm{mAb}$ A85-1 [PharMingen]; total IgG capture and detecting goat anti-mouse IgG [Southern Biotechnology Associates, Birmingham, Alabama, USA]), as described (26). The sensitivity of each immunoglobulin assay was $2 \mathrm{ng} / \mathrm{mL}$ for $\mathrm{IgE}, 0.16 \mathrm{ng} / \mathrm{mL}$ for $\mathrm{IgG}_{1}$, and $0.25 \mathrm{ng} / \mathrm{mL}$ for total IgG. The sensitivity of each cytokine assay was $0.31 \mathrm{ng} / \mathrm{mL}$ for IFN- $\gamma, 0.25 \mathrm{ng} / \mathrm{mL}$ for IL-2, $0.25 \mathrm{ng}$ for IL-10, and $8 \mathrm{pg} / \mathrm{mL}$ for IL-4. 
Assessment of GVHD. Transplanted animals were followed for more than 100 days after BMT. Body weights were measured on the day of transplantation, and then twice each week during the first month and once a week after that. Animals were also scored for clinical evidence of GVHD by assessment of changes in skin (i.e., alopecia, inflamed or scaly skin), generalized signs (fur texture, posture, and inflammation of the eyes), and diarrhea. Each parameter was quantified by scoring as follows: $0=$ normal, $1=$ mild, $2=$ moderate, $3=$ severe (for scoring of eye inflammation, 4 = eyes severely inflamed and closed).

Histopathology. For histopathological analysis of GVHD target tissues, samples were collected from large intestine, kidney, liver, and skin (from the head), and were fixed in $10 \%$ formalin. Formalin-preserved tissue samples were embedded in paraffin, cut into $5-\mu \mathrm{m}$ thick sections, and stained with hematoxylin and eosin for histological examination. Slides were coded without reference to mouse type or prior treatment status, and were systematically examined by a pathologist.

Statistical analysis. For comparison between groups of cytokine levels in supernatants, the Student's 2-tailed $t$ test for comparison of means was used. A $P$ value less than 0.05 was considered to be statistically significant. Survival data were analyzed using the Kaplan-Meier method of life table analysis, and statistical analysis was performed by the Mantel-Haenzsel test. Unless otherwise noted, data are presented as mean \pm SEM.

\section{Results}

Both Th1 cells and Th2 cells contribute to GVHD. To evaluate the separate contributions of Th1 and Th2 cells to the induction of GVHD in a fully MHC-mismatched and multiple minor histocompatibility antigen-mismatched strain combination, we compared the ability of BMC plus spleen cells from BALB/c-backcrossed $\mathrm{H}$ $2^{\mathrm{d}}$ STAT4 ${ }^{-/-}$mice, STAT6 ${ }^{-/-}$mice, or WT control BALB/c mice to induce GVHD in lethally irradiated C57BL/6 recipients. The number of donor $\mathrm{CD}^{+}$and $\mathrm{CD}^{+} \mathrm{T}$ cells administered in WT, STAT4-/-, and STAT6 ${ }^{-/-}$inocula was similar in all experiments, as measured by flow cytometry analysis of each inoculum. As shown in Figure 1 , the most severe mortality occurred in the group receiving WT splenocytes, which are capable of producing both Th1 and Th2 responses (median survival time $[\mathrm{MST}]=22$ days). STAT6 ${ }^{-/-} \mathrm{BMT}$, which is capable of producing high Th1 responses and no Th2 responses, induced mortality (MST $=31.5$ days) that was less rapid in onset than that induced by WT BMT $(P<$ 0.01). Mortality caused by the injection of STAT4 ${ }^{-/-}$ cells, which lack Th1 and produce enhanced Th2 responses, was consistently less rapid (MST $=79$ days) than that caused by both WT $(P<0.0001)$ and STAT6 ${ }^{-1-}$ cells $(P<0.001)$. Figure 1 shows the mortality of 117 animals from 6 separate experiments. We observed that the rapidity of onset of GVHD varied significantly from experiment to experiment, but in each experiment the GVHD mortality was most rapid when induced by WT cells, followed by STAT6 ${ }^{-/-}$cells and finally STAT4 ${ }^{-/-}$ cells. Furthermore, when GVHD was induced in the reverse strain combination, using $\mathrm{C} 57 \mathrm{BL} / 6$-backcrossed $\mathrm{H}-2^{\mathrm{b}} \mathrm{STAT4}^{-/}$, STAT6 ${ }^{--}$, or WT control $\mathrm{C} 57 \mathrm{BL} / 6$ mice to induce GVHD in lethally irradiated $\mathrm{BALB} / \mathrm{c}$ recipients, the same hierarchy of rapidity of GVHD mortality was observed: mortality induced by WT cells was the most rapid, followed by STAT6 ${ }^{-/}$cells and then STAT4 ${ }^{-/}$cells, the slowest (data not shown).

To further demonstrate that both Th1 cells and Th2 cells contribute to acute GVHD, we compared the severity and mortality of GVHD induced by STAT4 ${ }^{-/-}$cells, STAT6 ${ }^{-/-}$cells, and combined STAT4 ${ }^{-/-}$plus STAT6 ${ }^{-/-}$ cells. Mice received either a full dose $\left(13 \times 10^{6}\right)$ or a half dose $\left(6.5 \times 10^{6}\right)$ of both STAT4 ${ }^{-/-}$and STAT6 ${ }^{-/-}$spleen cells plus BMC, or $13 \times 10^{6} \mathrm{WT}_{\text {, STAT4 }}^{-/-}$, or STAT6 ${ }^{-/-}$ cells alone. Whereas recipients of the full dose of combined STAT4 ${ }^{--}$and STAT6 ${ }^{-/-}$spleen cells developed accelerated GVHD (MST $=6$ days) compared with recipients of WT inocula (MST $=16$ days), there was no difference in GVHD induced by $13 \times 10^{6} \mathrm{WT}$ spleen cells compared with the combination of $6.5 \times 10^{6}$ STAT4 $^{-/-}$ cells and $6.5 \times 10^{6}$ STAT6 $^{-/-}$cells $(\mathrm{MST}=15.5$ days $)$. Recipients of $13 \times 10^{6}$ STAT6 $^{-/-}$or STAT4 ${ }^{-/-}$spleen cells alone showed delayed GVHD compared with recipients of either $13 \times 10^{6} \mathrm{WT}$ cells or $6.5 \times 10^{6} \mathrm{STAT}^{-/-}$cells and $6.5 \times 10^{6}$ STAT6 $^{-/-}$cells, confirming that both Th1 cells and Th2 cells contribute to acute GVHD.

The addition of STAT4-/- cells to WT cells does not protect against GVHD. It has previously been shown that transplantation of polarized Th2 cells, after 7 days in culture with IL-2 and IL-4, reduced the GVHD mortality caused by nonpolarized cell populations (27). We therefore attempted to determine whether naive Th2 cells could ameliorate GVHD induced by Th1 cells by adding STAT4 $^{-/-}$BMC and spleen cells to WT cells. The addition of STAT4 ${ }^{--}$(Th2) cells to WT cells not only failed

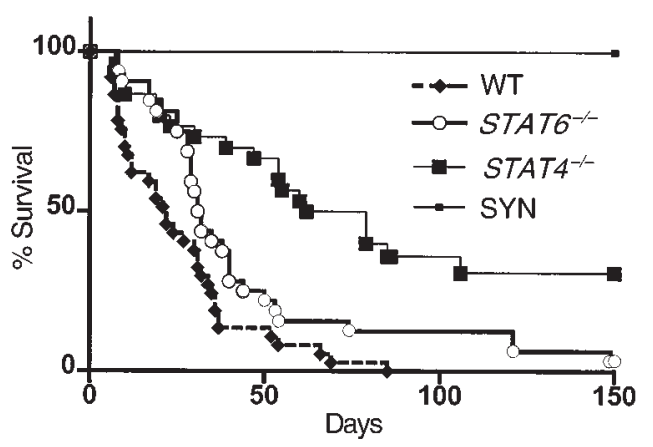

\section{Figure 1}

Both Th1 cells and Th2 cells contribute to GVHD mortality. Lethally irradiated B6 mice received $5 \times 10^{6} \mathrm{~T}$-cell-depleted $(\mathrm{TCD}) \mathrm{B} 6 \mathrm{BMC}$ and either syngeneic spleen cells (SYN) $(n=18)$, BALB/c WT BMC and spleen cells $(\mathrm{WT})(n=38)$, STAT4 ${ }^{-/-}$(Th2) BMC and spleen cells $(n=31)$, or STAT6 ${ }^{-1-}($ Th1 1$)$ BMC and spleen cells $(n=30)$. Each BALB/c (WT, STAT4 ${ }^{-/-}$, or STAT6 ${ }^{-/-}$) inoculum contained $13 \times 10^{6}$ spleen cells plus $10 \times 10^{6}$ BMC. Pooled data from 6 experiments, involving a total of 117 animals, are shown. 


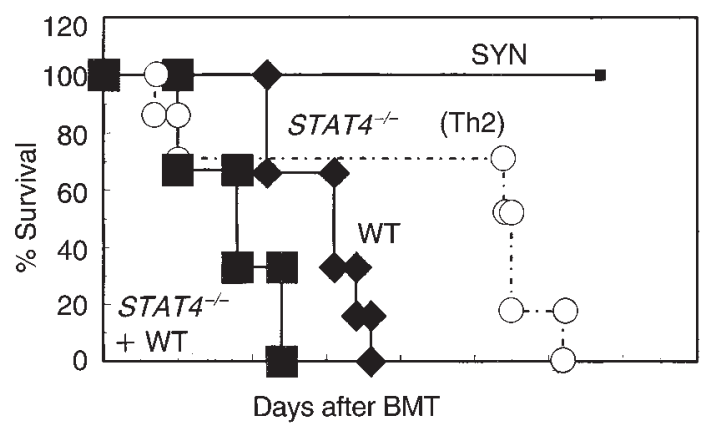

Figure 2

The addition of STAT4-/- cells to WT BMC plus spleen cell inocula does not protect from GVHD. Lethally irradiated $B 6$ mice received TCD B6 $\mathrm{BMC}$ and either syngeneic spleen cells $(n=3), \mathrm{BALB} / \mathrm{c} W T \mathrm{BMC}$ and spleen cells (WT) $(n=6)$, STAT4-/- (Th2) BMC and spleen cells $(n=8)$, or WT BMC and spleen cells plus STAT4 ${ }^{-1}$ BMC and spleen cells $\left(\right.$ STAT4 $\left.^{-1}+\mathrm{WT}\right)(n=3)$. Each inoculum contained $13 \times 10^{6} \mathrm{BALB} / \mathrm{c}$ spleen cells plus $10 \times 10^{6} \mathrm{BALB} / \mathrm{c}$ BMC (WT-induced control GVHD); $13 \times 10^{6} \mathrm{BALB} / \mathrm{c}$ STAT4 ${ }^{-/-}$spleen cells plus $10 \times 10^{6} \mathrm{BALB} / \mathrm{c}$ STAT4 ${ }^{-/-}$ BMC (STAT4 $4^{-/-}$-induced GVHD); or $6.5 \times 10^{6} \mathrm{BALB} / \mathrm{c} \mathrm{WT}$ spleen cells plus $6.5 \times 10^{6} \mathrm{BALB} / \mathrm{c} \mathrm{STAT4}^{-/-}$spleen cells plus $5 \times 10^{6} \mathrm{BALB} / \mathrm{cWT}$ BMC plus $5 \times 10^{6} \mathrm{STAT4}^{-/-} \mathrm{BMC}\left(\mathrm{STAT4}^{-/-}+\mathrm{WT}\right.$ cells). Results of one of two similar experiments are shown.

to prevent or reduce the severity of acute GVHD (MST $=18$ days), but it accelerated GVHD mortality compared with that induced by WT cells alone (MST $=31$ days) (Figure 2 shows one of two similar experiments). This result further emphasizes that Th2 cells play a pathogenic role in GVHD, and shows that they do not effectively inhibit GVHD mediated by naive cells that can differentiate into Th1 cells.

Unique clinical and pathological syndromes associated with Th2 and Th1 GVHD. We monitored transplanted animals for clinical signs of GVHD. Figure 3 shows the mortality curve and weight curve from a representative experiment. GVHD scores for generalized signs (hunching, ruffled fur, and periorbital edema) of WT recipients were the most severe in intensity and were the earliest in onset: on day 8, mean GVHD score was $9.8 \pm 0.6$ out of a possible maximum score of 10 . The recipients of STAT6 ${ }^{-{ }^{-}}$BMT and STAT4 ${ }^{-{ }^{-}}$BMT had GVHD scores that were lower at this time point (on day 8 , mean score for recipients of STAT4 ${ }^{-/}$BMT was $2.3 \pm$ 1.2 , and for recipients of $S T A T 6^{-/-}$BMT it was $5.4 \pm 0.5$ ) and were similar to each other. However, the time required to achieve the maximal score for generalized signs of GVHD was earlier in recipients of STAT6 ${ }^{-1}$ BMT (on day 12, mean score was $6.7 \pm 0.6$ ) than in STAT4 ${ }^{-/-}$BMT recipients (on day 16, mean score was 7 $\pm 1)$. A weight curve, which provides a sensitive indicator of GVHD, is shown in the lower panel of Figure 3. Animals receiving WT cells rapidly lost weight before their deaths by day 13. Animals receiving Th1 $\left(\right.$ STAT6 $\left.^{-/}\right)$and Th2 (STAT4-/-) BMT showed initial weight loss during the first 10 days after BMT. However, the clinical signs subsequently diverged markedly in the two groups. Whereas recipients of STAT6 $6^{--}$BMT developed severe diarrhea and began to show mortality around day 25 , recipients of STAT4-/- BMT did not have diarrhea, and had only mild weight loss. As shown in the upper panel of Figure 3, recipients of STAT4 $4^{--}$ BMT began to show mortality around day 30 . These mice developed extremely severe skin GVHD, which began around the eyes, mouth, and ears and progressed to involve the whole body, resulting in hair loss and profound scaling. This striking clinical picture was observed in all six experiments, with time of onset ranging from 15 to 50 days after BMT. Whereas these clinical changes were not observed in any experiments in the animals that received BMT from WT or STAT6- ${ }^{--}$ mice and survived until day 100 , they were present in the majority (11 of 14 ) of STAT4-/- BMT recipients that survived to 10 weeks after BMT.

Different localization of pathological GVHD-associated changes in recipients of WT, STAT4-/-, and STAT6-/- BMT. After death, carcasses were fixed in formaldehyde and the tissues were processed and scored in a blinded fashion for lesions associated with GVHD. All analyzed organs were harvested from animals that died at similar times after transplantation from two experiments where the severity of GVHD was similar (MSTs in days for experiment 1 and 2: WT $=12$ and 19, STAT6 ${ }^{--}=35$ and 29, STAT4 $^{-/}=79$ and 155). The liver, spleen, lungs, kidney, and large intestine were examined. There were
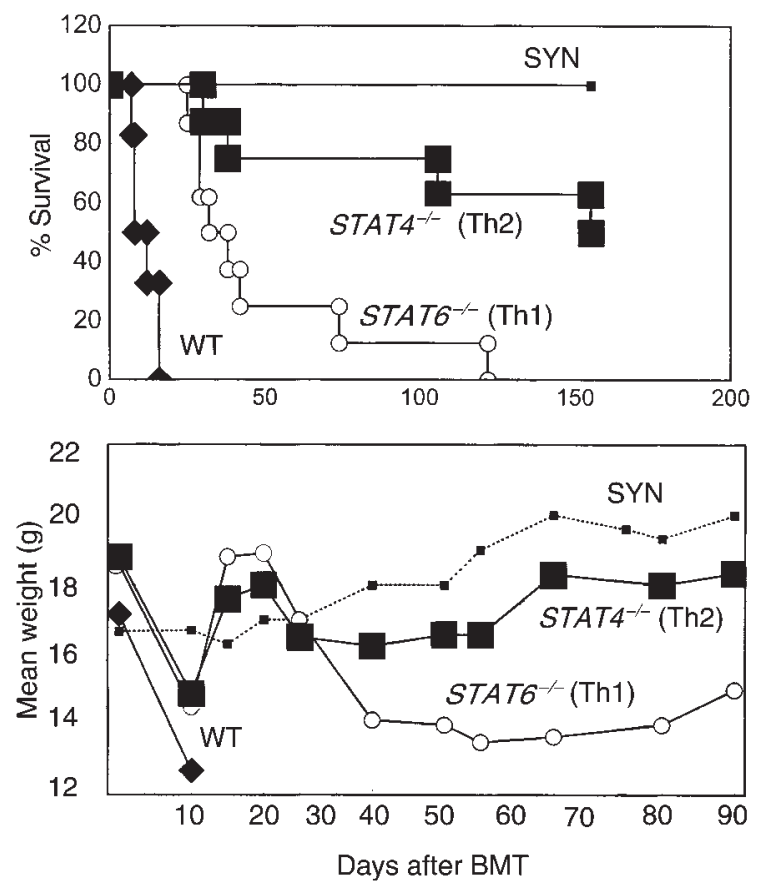

Figure 3

Weight loss and mortality for WT-, Th1-, and Th2-mediated GVHD in a single representative experiment. GVHD mortality is shown in the upper panel, and GVHD-induced weight loss is shown in the lower panel. Lethally irradiated B6 mice received $5 \times 10^{6} \mathrm{TCD}$ B6 BMC and either syngeneic spleen cells $(n=3), \mathrm{BALB} / \mathrm{c}$ WT BMC and spleen cells (WT) $(n=6)$, STAT4-/- (Th2) BMC and spleen cells $(n=8)$, or STAT6-/(Th1) BMC and spleen cells $(n=8)$. Each BALB/c (WT, STAT4 ${ }^{-/}$, or STAT6 ${ }^{-/-}$) inoculum contained $13 \times 10^{6}$ spleen cells plus $10 \times 10^{6} \mathrm{BMC}$. 


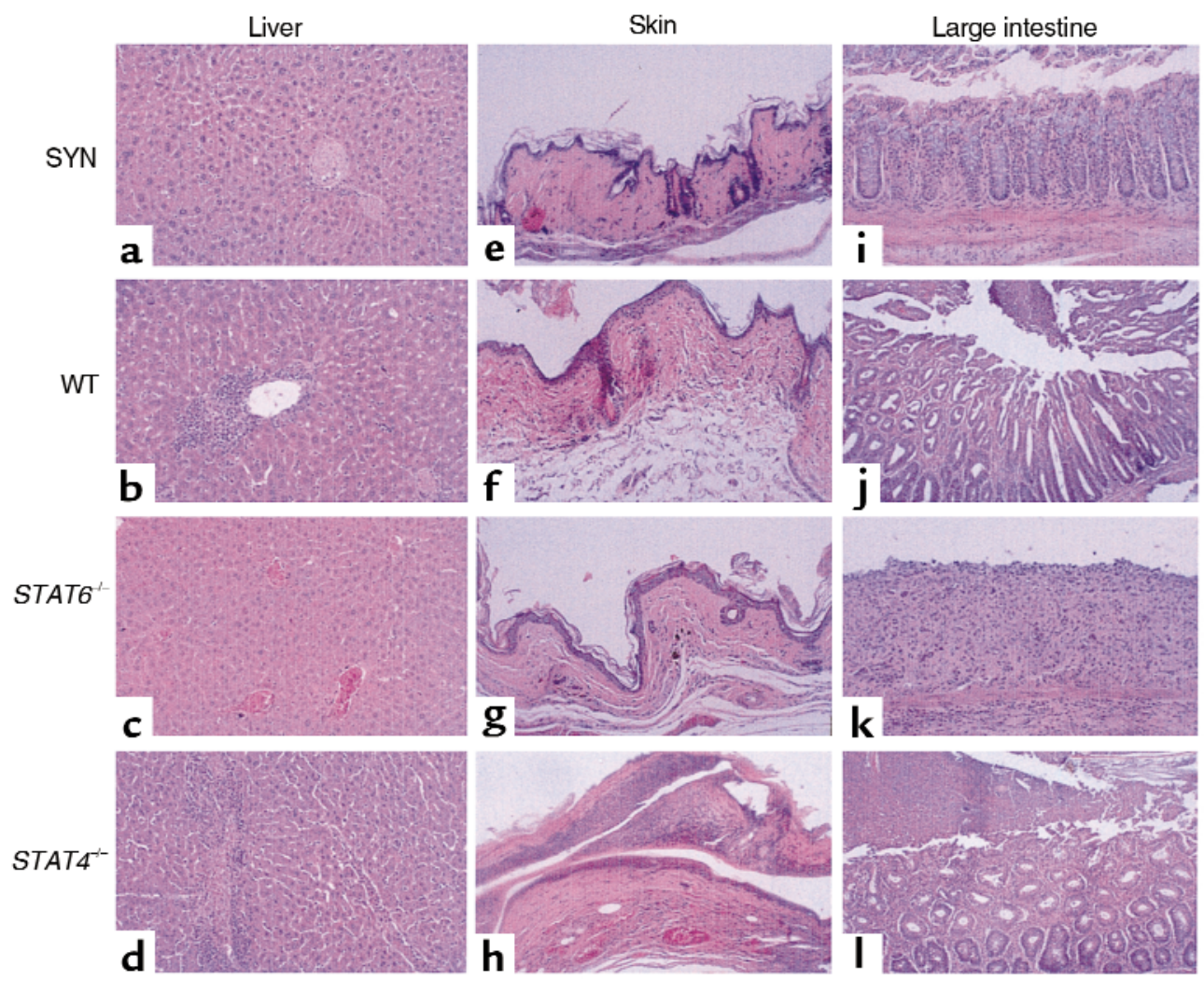

\section{Figure 4}

Histological evidence of GVHD in the liver, skin, and large intestine. Liver, skin, and large intestine sections were obtained from lethally irradiated B6 recipients reconstituted with syngeneic BMT (a, e, i), WT BMT $(\mathbf{b}, \mathbf{f}, \mathbf{j}), \mathrm{STAT6}^{-/-} \mathrm{BMT}(\mathbf{c}, \mathbf{g}, \mathbf{k})$, or STAT4 ${ }^{-/-}$BMT (d, h, I). Lethally irradiated B6 mice received $5 \times 10^{6} \mathrm{TCD}$ B6 BMC and either syngeneic spleen cells, BALB/c WT BMC and spleen cells (WT), STAT4 ${ }^{-/}$(Th2) BMC and spleen cells, or STAT6 ${ }^{-/-}$(Th1) BMC and spleen cells. Each BALB/c (WT, STAT4-/-, or STAT6 ${ }^{-/-}$) inoculum contained $13 \times 10^{6}$ spleen cells plus $10 \times 10^{6}$ $B M C$. These samples, with the exception of those from recipients of syngeneic BMT, were harvested from animals that died during the third week after BMT from two experiments in which the severity of GVHD was similar. Animals that died at later time points showed similar pathological changes. Syngeneic BMT recipients were sacrificed 200 days after BMT. Photographs were taken through a $\times 50$ objective lens.

no pathological changes in the appearance of liver (Figure 4a), skin (Figure 4e), or large intestine (Figure 4i) in recipients of syngeneic BMT. Moderate to severe GVHD-induced liver changes were observed in 5 of 5 recipients of WT BMT, which died on days 19, 24, 27, and 54 after BMT. Figure $4 \mathrm{~b}$ shows typical liver lesions found in these mice; portal areas were diffusely infiltrated by a mixed population of leukocytes including neutrophils, lymphocytes, macrophages, and a few plasma cells. There was associated multifocal, singlecell hepatocellular necrosis, consistent with severe hepatic GVHD. Similar changes were observed in 4 of 4 recipients of $\mathrm{STAT4}^{-/-} \mathrm{BMT}$, which died on days 20, 39, 47, and 106 after BMT (Figure 4d). In striking contrast, liver sections of $\mathrm{STAT}^{-1-} \mathrm{BMT}$ recipients that died at similar times $(22,23,25,26,27,29$, and 38 days after BMT) appeared normal, without any infiltrate or evidence of cholestasis (Figure 4c).

All recipients of WT BMT showed signs of slight skin hyperkeratosis. In some mice ( 3 of 7 ) that died on days 19 and 27 after BMT, there were very mild focal mononuclear inflammatory cell infiltrates located mostly in the dermis (Figure 4f). In accordance with the clinical picture, recipients of STAT4 ${ }^{-/}$BMT (3 of 4 ) that died on days 20,39, and 106 after BMT showed marked skin abnormalities. In some animals, pustules and crusting with neutrophil infiltration was observed on the skin's surface (Figure 4h). In all animals, the dermis was infiltrated by a mixed population of leukocytes, including lymphocytes, macrophages, and neutrophils (Figure 4h). In several places the cells formed granulomas. There was also a suggestion of lysis along the epidermal-dermal junction. Consistent with the clinical picture, inflammatory changes were absent in all 12 recipients of STAT6 $6^{-1-}$ BMT that died on days $5,22,23$, $25,26,27,29,38$, and 44 after BMT (Figure 4g). Furthermore, these severe skin changes were not observed in mice with GVHD induced by STAT6 $6^{-1}$ that had longterm survival (4 animals that died at 74, 122, 149, and 150 days after BMT). Their skin showed signs of mild hyperkeratosis, but without inflammatory changes.

Recipients of WT and STAT6 ${ }^{-1}$ BMT showed clinical signs of severe colitis manifested by profuse diarrhea and weight loss. The pathological examination revealed that in 7 of 7 recipients of WT inocula, which died on days 19, 24, 27, 39, and 54 after BMT, there was a thick- 
$\mathbf{a}$

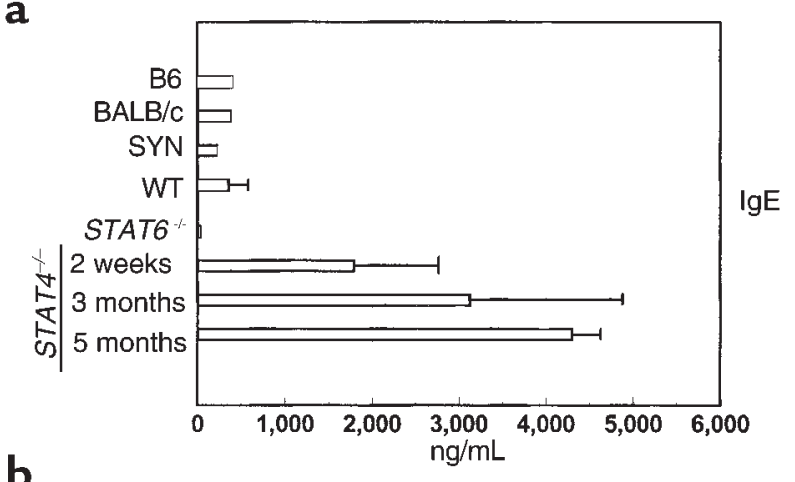

b

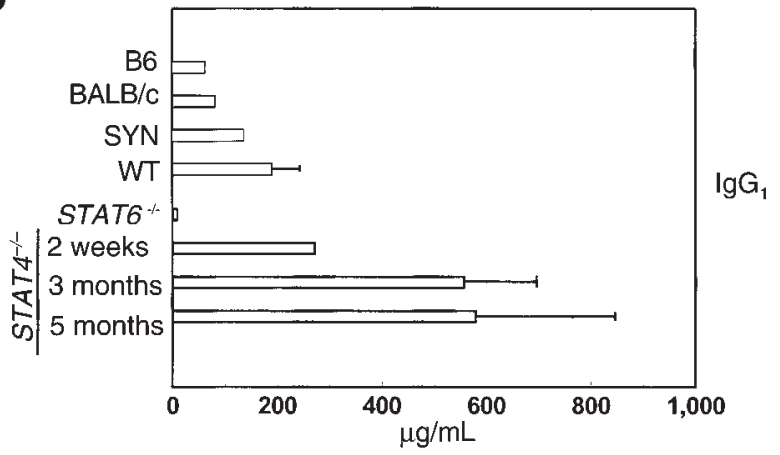

\section{Figure 5}

Serum $\lg E(\mathbf{a})$ and $\lg \mathrm{G}_{1}(\mathbf{b})$ levels in GVHD induced by WT, STAT6-1-, and STAT4 ${ }^{-1-}$ cells. C57BL/ 6 animals were transplanted with syngeneic, WT BALB/c, STAT4 ${ }^{-/-}$, or STAT6 ${ }^{-/-}$cells. Lethally irradiated B6 mice received $5 \times 10^{6} \mathrm{TCD} B 6 \mathrm{BMC}$ and either syngeneic spleen cells, BALB/c WT BMC and spleen cells (WT), STAT4 ${ }^{-/-}$(Th2) BMC and spleen cells, or STAT6 $6^{-1-}$ (Th1) BMC and spleen cells. Each BALB/C (WT, STAT4-/-, or STAT6 $\left.{ }^{-/-}\right)$inoculum contained $13 \times 10^{6}$ spleen cells plus $10 \times 10^{6} \mathrm{BMC}$. For syngeneic BMT and for WT- and

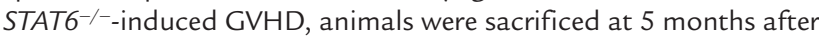
BMT. Recipients of STAT4 ${ }^{-/-}$cells were bled and sacrificed at 2 weeks, at 3 months, and at 5 months after BMT. Sera were collected and Ig levels were measured by ELISA. For each time point for each group, 2-4 animals were analyzed. MSTs in days for this experiment were 26 for recipients of WT cells, 54 for recipients of STAT4 ${ }^{-/}$cells, and 35 for recipients of STAT6 ${ }^{-/}$cells. Concentrations of IgE and $\lg G_{1}$ in sera are expressed as mean \pm SEM.

ening of colonic mucosa with mild to severe infiltration of mononuclear inflammatory cells. The epithelium was hyperplastic with elongated, tortuous, and branched crypts (Figure 4j). The colon sections of recipients of STAT6 $6^{-1}$ BMT showed similar changes in 9 of 9 examined animals, which died on days $5,22,23,25$, $26,27,29,38$, and 44 after BMT. Three of 9 animals had extremely severe chronic colitis, with lumens that were filled with exudate and severely ulcerated mucosa (Figure $4 \mathrm{k}$ ). The changes observed in recipients of STAT6 ${ }^{-/}$BMT were more severe than changes observed in recipients of WT BMT.

Although the recipients of STAT4-/- BMT lacked clinical signs of colitis or weight loss, in 3 of 4 animals that died on days 39,47 , and 106 after BMT, there were histological signs of colitis, with a thickening of the mucosa and diffuse cellular infiltrate (Figure 4l). How- ever, these changes were less severe than those observed in recipients of WT or STAT6 ${ }^{-1-}$ BMT.

There were no pathological changes in the appearance of lungs or kidneys in any of the 3 groups of recipients (not shown).

STAT6 ${ }^{--}$cells and STAT4 $4^{--}$cells exbibit stable Th1 and Th2 phenotypes, respectively, in vivo. Cytokines modulate B-cell function by differentially stimulating specific Ig isotype production. Th1 responses are characterized by an increase in the level of $\operatorname{IgG}_{2 a}$ production (2). Similarly, immune responses characterized by high levels of IL-4 lead to elevated serum $\operatorname{IgE}$ and $\mathrm{IgG}_{1}$, and suppress the production of $\operatorname{IgG}_{2 \mathrm{a}}(2)$. Increases in serum $\operatorname{IgE}$ have been reported in GVHD in man and mouse $(28,29)$. We followed serum IgE levels in the GVHD mice over time. Normal nontransplanted C57BL/6 and BALB/c ageand sex-matched mice contained an average of $300 \mathrm{ng} / \mathrm{mL} \operatorname{IgE}$ in their sera at the age of 20 weeks. $\operatorname{IgE}$ levels in sera of the WT and syngeneic BMT recipients did not differ significantly from those of controls (Figure 5a). In contrast, the recipients of STAT4-/BMT-induced GVHD showed significantly increased serum IgE levels as early as 2 weeks after BMT (Figure $5 a)$. These levels increased over time, becoming greater than $4,000 \mathrm{ng} / \mathrm{mL}$ in GVHD mice sacrificed 5 months after BMT. The recipients of STAT6 ${ }^{-/-}$BMT showed almost no detectable IgE in the serum at the time of sacrifice 5 months after BMT (Figure 5a). This profile of high levels of IgE in STAT4 ${ }^{-/-}$BMT-induced GVHD, and IgE deficiency in the recipients of STAT6 $6^{-1-}$ BMT, indicates that donor-derived $\mathrm{T}$ cells retain their Th1 or Th2 phenotype in GVHD mice.

We also followed the $\operatorname{IgG}_{1}$ levels in the recipients of STAT4 ${ }^{--}, \mathrm{STAT6}^{-/}$, WT, and syngeneic BMT. The levels of $\mathrm{IgG}_{1}$ in sera of these animals followed the IgE pattern. The recipients of STAT4-/- GVHD inocula had significantly higher levels of $\operatorname{IgG}_{1}$ in their sera than did the recipients of WT, $S T A T 6^{--}$, or syngeneic inocula, and this level increased over time (Figure 5b). In contrast, the recipients of $S T A T 6^{-/-}$GVHD inocula had almost undetectable levels of serum $\operatorname{IgG}_{1}$ (Figure $5 \mathrm{~b}$ ). Total IgG levels were similar in the recipients of STAT6-/$\operatorname{BMT}(312.9 \pm 152.0 \mu \mathrm{g} / \mathrm{mL})$ sacrificed at 5 months after BMT and in recipients of STAT4-/- cells (470.3 \pm $38.0 \mu \mathrm{g} / \mathrm{mL}$ ) sacrificed at 2 weeks after BMT, despite the difference in $I_{g G}$ levels cited above.

Donor engraftment and Thelper phenotype in recipients of WT, STAT4 $4^{--}$, and STAT6 $6^{--}$BMT. Recipients of STAT4 $4^{-/}$cells were sacrificed at 2 weeks, 3 months (87 days), and 5 months (136 days) after BMT. Recipients of syngeneic and WT cells were sacrificed at 2 weeks and at 3 months (87 days) after BMT. For STAT6-/-induced GVHD, animals were sacrificed only at 5 months (136 days) after BMT. MLCs were prepared with host-type (B6) irradiated stimulator cells. FACS ${ }^{\circledR}$ analysis of peripheral blood lymphocytes and bone marrow showed that these animals were full donor-type hematopoietic chimeras at all 3 time points (data not shown). The splenocytes from these animals showed mixed lymphocyte response reactivity both toward 

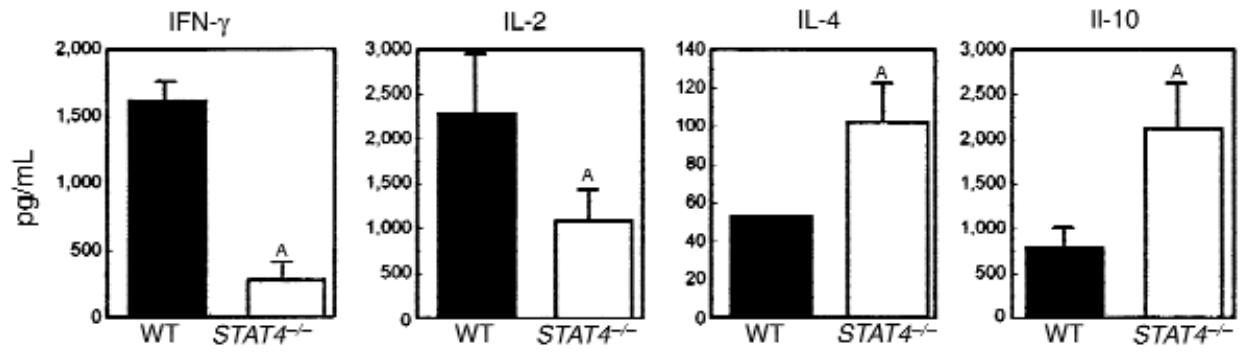

\section{Figure 6}

Cytokine profile of splenocytes from mice with WT- or STAT4-/--induced GVHD. Lethally irradiated C57BL/ 6 host mice transplanted with 5 $\times 10^{6} \mathrm{TCD}$ B6 BMC and either $13 \times 10^{6} \mathrm{WT}$ spleen cells plus $10 \times 10^{6} \mathrm{WT} \mathrm{BMC}(n=2)$ or $13 \times 10^{6}$ STAT4 $4^{-/-}$spleen cells plus $10 \times 10^{6}$ STAT4 ${ }^{-/}$BMC $(n=4)$ were sacrificed at 2 weeks after BMT, and splenocyte suspensions were prepared. Their donor-derived phenotype was demonstrated by FACS ${ }^{\circledR}$. The cells were cultured with irradiated splenocytes from C57BL/ 6 mice. Supernatants were harvested 72 hours after initiation of MLC, and cytokine levels were determined by ELISA. MSTs for this experiment were 26 days for recipients of WT cells and 54 days for recipients of STAT4 ${ }^{-/}$cells. Concentrations of IFN- $\gamma$, IL-2, IL-4, and IL-10 in cell culture supernatants are expressed as mean \pm $\operatorname{SEM}(\mathrm{pg} / \mathrm{mL}) .{ }^{A} P<0.01$ vs. WT group.

host C57BL/6 cells and third-party strain A/J, while being nonreactive toward donor antigens (data not shown). Thus, there was constant, ongoing activation and proliferation of donor $\mathrm{T}$ cells toward host antigens. By quantifying the levels of IFN- $\gamma$, IL-2, IL-4, and IL-10 in MLC supernatants from WT and STAT4-- BMT recipients, distinct patterns emerged. In supernatants obtained from spleen cells of mice receiving STAT4-deficient cells, the amounts of IL-4 and IL-10 (i.e., the Th2-specific cytokines) were increased compared with those in supernatants of spleen cells from WT recipients (see Figure 6). Similar results were obtained at all 3 time points after BMT. IFN- $\gamma$ and IL-2, which are both induced during the Th1 response, were detected at higher levels in supernatants of spleen cells from recipients of WT BMT than in recipients of STAT4 ${ }^{-/}$ BMT (see Figure 6). Although produced at lower levels than in the recipients of WT BMT, there was measurable production of IFN- $\gamma$ in the recipients of STAT4 ${ }^{-1-}$ BMT (see Figure 6). Due to profound atrophy of the spleen at 5 months after BMT, only 1 recipient of STAT6 ${ }^{-/}$BMT was available for analysis. This mouse showed the expected Th1 phenotype, with large amounts of IFN- $\gamma(781 \mathrm{pg} / \mathrm{mL})$ and IL-2 $(591 \mathrm{pg} / \mathrm{mL})$, low amounts of IL-10 $(228 \mathrm{pg} / \mathrm{mL})$, and undetectable IL-4 in the collected supernatants.

\section{Discussion}

Our study indicates that the role of Th1 cells and Th2 cells in GVHD is more complicated than previously thought. We have shown, contrary to other studies $(6$, $8,27,30)$, that both Th1 cells and Th2 cells contribute to acute GVHD. However, each subset causes injury of specific tissues, resulting in a unique clinical picture when Th1 or Th 2 responses separately induce GVHD. Most strikingly, Th2 cells appear to be absolutely required for the induction of hepatic GVHD and severe skin GVHD. If confirmed in humans, these observations could have important implications for the therapy of GVHD, because they suggest that Th2targeted approaches might be used to most effectively and specifically target liver and skin manifestations of acute GVHD, which are frequently seen without intestinal GVHD in clinical BMT.

We consistently observed that WT cells induced the most severe GVHD when compared with that induced by STAT6 $6^{-/-}$(Th1) or STAT4 $4^{--}$(Th2) cells. In other studies, we have observed that GVHD induced by $I L$ $4^{-/-}$cells (wherein the disease results mostly from Th1 cells, but with some Th2 component due to residual signaling through IL-13) was of a severity intermediate between that induced by WT cells and STAT6-/cells (B. Nikolic and M. Sykes, unpublished data). In further support of the hypothesis that both Th1 cells and Th2 cells contribute to GVHD, our group and others have shown that acute GVHD can occur in the absence of IFN- $\gamma(11,12)$, and that GVHD induced by donors that cannot produce IL-4 is milder than that induced by WT cells (12). Furthermore, the administration of anti-IL-4 mAb can ameliorate acute GVHD (31). Previous studies have shown that systemic administration of the Th1-inducing or Th1-associated cytokines IL-2 (18), IL-12 (32), or IFN- $\gamma(33)$ can inhibit GVHD. Paradoxically, in other models, the administration of anti-IL-2 or anti-IL-12 mAbs (31, 34) has also been shown to prevent acute GVHD. Apparently, cytokines play complex roles in the induction and inhibition of GVHD, and the timing, the route of administration, and the strain combination may be critical in determining their effect $(11,35-38)$.

Although the experiments described herein were performed under similar conditions, with careful matching of the ages of donors and recipients and of the number of injected cells and dose of irradiation, we have always observed wide variability in the severity of GVHD from experiment to experiment, particularly over long time periods. There seem to be periods of months or years in which a given T-cell dose produces GVHD of a certain tempo in a particular model, and then this tempo later changes for reasons that cannot be identified. To minimize the effect of this variability and enable interpretation of data, we included every control group in every 
experiment. GVHD induced by WT cells serves as a standard for the severity of GVHD, and syngeneic BMT acts as a negative control. In this study, we consistently observed that GVHD mortality was more rapid when induced by cells from STAT6 ${ }^{-/-}$mice than that induced by STAT4 ${ }^{-/}$cells. Nevertheless, the observed variability in the severity of GVHD and differences between human and murine GVHD mandate additional studies before direct translation of these results to human transplantation should be considered.

Although our results are at variance with previous studies suggesting that Th2 cells do not cause, and even prevent acute GVHD, these studies used spontaneous or LPS-induced mortality as an endpoint, and did not show an absence of GVHD pathology in recipients of Th2 cells $(8,27)$. Another possible reason for the inconsistency of our results with others that implicated Th1 cells but not Th2 cells in GVHD is that some of the previous studies relied on polarization of $\mathrm{T}$ helper cells by incubation with cytokines for several days in vitro $(5,8$, 27). Polarized T cells were then transferred to irradiated recipients. This procedure might result in incomplete polarization, partial anergy, or a failure of subsequent proliferation, and could allow secondary conversion of $\mathrm{T}$ cells to a mixed phenotype upon transfer to BMT recipients. Indeed, examination of T-cell subsets in these BMT models revealed that T-cell populations were not polarized into "classical" Th1 or Th2 cytokine phenotypes 2 weeks after BMT (8), and that intermediate cytokine profiles of both $\mathrm{CD}^{+}$and $\mathrm{CD} 4^{+} \mathrm{T}$-cell subsets developed, as has been described in other systems (30). STAT4- and STAT6-deficient mice have the advantage over these experimental models of allowing more pure Th1 or Th2 responses to be examined, and of not requiring a priming step before $T$ cells are transferred for the induction of GVHD $(15,17,39,40)$. Although STAT6 ${ }^{-/-}$lymphocytes are completely impaired in their ability to generate IL-4-secreting cells, we were able to detect some IFN- $\gamma$ in supernatants of MLC from STAT4-/-induced GVHD (Figure 6). A recent study showed that under conditions that promote Th1 cell differentiation, it is possible to detect low levels of production of IFN- $\gamma$ via a STAT4-independent pathway (41). However, whereas mice that are both STAT4 ${ }^{--}$and STAT6 ${ }^{-/-}$are able to produce significant amounts of IFN- $\gamma$ and mount Th1 cell-mediated delayed-type hypersensitivity, STAT4 ${ }^{-/-}$mice retain their predominantly Th2 phenotype, presumably through dominant IL-4 activation of STAT 6 or Th2-mediated inhibition of Th1 cytokine production, or both (41).

Perhaps in keeping with our data showing that Th1 cells are associated with severe intestinal GVHD, a requirement for Th1 responses in inducing pathology in 2 different models of experimental colitis was recently demonstrated (42). Treatment with anti-IL-12 Abs or bone marrow reconstitution of $\mathrm{T}$ cell- and NK cell-deficient Tge26 mice from STAT4-deficient mice resulted in modulation of the severity of disease (42). Thus, the reduced intestinal pathology in Th2 recipi- ents may have been an important determinant in the observed prolonged survival of recipients of STAT4 ${ }^{-1-}$ BMT. This may also explain data from other experiments in which mortality of recipients of in vitro-polarized Th2 cells was diminished or even absent $(5,7,8,27)$. In some of these studies, models were used that tend to induce milder GVHD, perhaps accounting for the failure to observe the contribution of Th2 cells to acute GVHD. Consistent with the failure of Th2 cells to induce acute GVHD across minor histocompatibility barriers without $\mathrm{MHC}$ differences (8), a recent study demonstrated that Th2 cells were unable to reject MHC-matched, minor histocompatibility antigen-mismatched cells, but were able to rapidly reject fully MHC-mismatched grafts (43). Thus, the presence of full MHC disparities in our model may partially explain the apparent discrepancy with the results of Krenger et al. $(8,27)$.

Consistent with our data showing a requirement for Th2 cells in hepatic and skin GVHD, a recent study has shown that although mortality from GVHD is substantially diminished after infusion with donor in vitro-polarized Th2 cells, clinical signs of GVHD are still present in surviving mice, and target organ pathology persists. Significant cutaneous and hepatic histopathological damage was evident 7 weeks after BMT in mice receiving polarized donor Th2 cells, and was equivalent to that in animals receiving naive donor T cells (8). Furthermore, after induction of GVHD with $I F N-\gamma^{-1-}$ cells, extensive mononuclear cell infiltrates were seen in livers (12); treatment with anti-IL-4 mAb markedly reduced $\mathrm{IgE}$ and $\mathrm{IgG}_{1}$ levels, and liver disease was ameliorated (31). Increased levels of IgE correlated strongly with the histological grades and stages of liver disease in parental mice receiving $F_{1}$ spleen cell transfer, and treatment with anti-IL-4 mAb prevented both phenomena (44). In humans, predominant activation of Th2 cells, indirectly measured by elevated serum IgE levels, was associated with liver disease in GVHD (28).

$\mathrm{T} 1(\mathrm{Tc} 1)$ and T2 (Tc2) subsets of CD8 ${ }^{+} \mathrm{T}$ cells secrete polarized patterns of cytokines similar to the Th1 and Th2 CD 4 subsets. Although both T1 and T2 CD8 ${ }^{+} \mathrm{T}$ cells exhibit comparable levels of perforin-mediated cytolysis (45), T1 CD8 ${ }^{+} \mathrm{T}$ cells exhibit significantly higher Fas-mediated cytolysis (45). Fas-mediated anti-recipient cytotoxicity has been implicated in the development of hepatic and cutaneous GVHD against minor histocompatibility antigens (46), but this was not reproduced in a full haplotype-mismatched strain combination (47). In another study, administration of an anti-Fas ligand inhibitory $\mathrm{mAb}$ resulted in a reduction of the weight loss and mortality associated with GVHD directed against a full haplotype mismatch, but without improvement in skin or liver lesions (48). Therefore, Tc1-mediated cytolysis may not play a major role in hepatic and skin GVHD caused by a full MHC disparity. In accordance with these data, we have observed that only Th2 and Tc2 cells induced liver and skin GVHD in a fully MHC-mismatched strain combination. 
The preferential injury of gut-associated tissue in Th1-induced GVHD, and of skin and liver in GVHD caused by Th 2 cells, may be the result of different homing properties of the different subsets. Indeed, there is growing evidence that in addition to effector function, activated Th1 and Th2 lymphocytes acquire different migratory capacities (49-52). Chemokines and chemokine receptors are probably important for the selective migration of T-cell subsets (50). Distinct profiles of chemokine receptors are acquired by $\mathrm{T}$ cells after polarization; these are modulated by cytokines (50-52). Indeed, in vivo studies have confirmed this concept. Mouse Th1 cells express the functional ligand for P-selectin and E-selectin, PSGL-1, which promotes their migration into inflamed tissue (53). It was recently shown that Th1 cells could efficiently enter inflamed sites, such as sensitized skin or arthritic joints (53). In our model, preferential migration of Th2 cells, but not Th1 cells, into the liver and skin was observed, suggesting that other receptors or chemokines may play a decisive role in this migratory process.

There are potential dangers associated with therapies based on immune deviation from Th1 responses to Th2 responses. Th2 responses are responsible for the pathology of allergic diseases and at least 1 autoimmune condition (54-57). Our data indicate that manipulation of the Th1/Th2 balance as an approach to the prevention of GVHD may not be ideal. Possible severe side effects of such treatment should be considered before proceeding with human trials. However, our results suggest that the potential to treat hepatic and skin GVHD with anti-Th2 cytokine therapy deserves exploration.

\section{Acknowledgments}

This work was supported by National Institutes of Health (NIH) Grant HL-49915. B. Nikolic was supported in part by The Daland Fellowship for Research in Clinical Medicine (American Philosophical Society) and by an American Society of Transplant Physician-Sandoz Fellowship in Transplantation Award. M.J. Grusby was supported by NIH Grant AI-40171 and a gift from The Mathers Foundation. M.J. Grusby is a scholar of The Leukemia Society of America. S. Lee is currently studying at the Division of Clinical Immunology, Huddinge University Hospital, Karolinska Institute, Sweden. We thank Diane Plemenos for assistance in preparing the manuscript. We also thank David Anderson, Henry Winn, and Hugh Auchincloss for helpful review of the manuscript. We thank David H. Sachs for helpful advice.

1. Butturini, A., and Gale, R.P. 1988. T cell depletion in bone marrow transplantation for leukemia: current results and future directions. Bone Marrow Transplant. 3:185-192.

2. Abbas, A.K., Murphy, K.M., and Sher, A. 1996. Functional diversity of helper T lymphocytes. Nature. 383:787-793.

3. Antin, J.H., and Ferrara, J.L.M. 1992. Cytokine dysregulation in acute graft-versus-host disease. Blood. 80:2964-2968.

4. Blazar, B.R., Korngold, R., and Vallera, D.A. 1997. Recent advances in graft-versus-host disease (GVHD) prevention. Immunol. Rev. 157:79-109.

5. Fowler, D.H., Kurasawa, K., Smith, R., Eckhaus, M.A., and Gress, R.E. 1994. Donor CD4-enriched cells of Th2 cytokine phenotype regulate graft-versus-host disease without impairing allogeneic engraftment in sublethally irradiated mice. Blood. 84:3540-3549.

6. Fowler, D.H., Kurasawa, K., Husebekk, A., Cohen, P.A., and Gress, R.E. 1994. Cells of the Th2 cytokine phenotype prevent LPS-induced lethality during murine graft-versus-host reaction. Regulation of cytokines and CD8+ lymphoid engraftment. J. Immunol. 152:1004-1013.

7. Pan, L., Delmonte, J., Jalonen, C.K., and Ferrara, J.L.M. 1997. Pretreatment of donor mice with granulocyte colony-stimulating factor polarizes donor $\mathrm{T}$ lymphocytes toward type- 2 cytokine production and reduces severity of experimental graft-versus-host disease. Blood. 86:4422-4429.

8. Krenger, W., et al. 1996. Transplantation of polarized type 2 donor T cells reduces mortality caused by experimental graft-versus-host disease. Transplantation. 62:1278-1285.

9. Blazar, B.R., Taylor, P.A., Smith, S., and Vallera, D.A. 1995. Interleukin10 administration decreases survival in murine recipients of major histocompatibility complex disparate donor bone marrow cells. Blood. 85:842-851.

10. Krenger, W., Snyder, K., Smith, S., and Ferrara, J.L.M. 1994. Effects of exogenous interleukin-10 in a murine model of graft-versus-host disease to minor histocompatibility antigens. Transplantation. 58:1251-1257.

11. Yang, Y.-G., Dey, B., Sergio, J.J., Pearson, D.A., and Sykes, M. 1998. Donor-derived interferon $\gamma$ is required for inhibition of acute graft-versus-host disease by interleukin 12. J. Clin. Invest. 102:2126-2135.

12. Murphy, W.J., et al. 1998. Differential effects of the absence of interferon- $\gamma$ and IL-4 in acute graft-versus-host disease after allogeneic bone marrow transplantation in mice. J. Clin. Invest. 102:1742-1748.

13. Kaplan, M.H., Sun, Y.-L., Hoey, T., and Grusby, M.J. 1997. Impaired IL12 responses and enhanced development of Th2 cells in STAT4-deficient mice. Nature. 382:174-177.

14. Thierfelder, W.E., et al. 1996. Requirement for STAT4 in interleukin-12mediated responses of natural killer and T cells. Nature. 382:171-174.

15. Takeda, K., et al. 1996. Essential role of STAT6 in IL-4 signalling. Nature. 380:627-630.

16. Kaplan, M.H., Schindler, U., Smiley, S.T., and Grusby, M.J. 1996. STAT6 is required for mediating responses to IL-4 and for the development of Th2 cells. Immunity. 4:313-319.

17. Shimoda, K., et al. 1996. Lack of IL-4-induced Th2 response and IgE class switching in mice with disrupted STAT6 gene. Nature. 380:630-633.

18. Sykes, M., Romick, M.L., and Sachs, D.H. 1990. Interleukin 2 prevents graft-versus-host disease while preserving the graft-versus-leukemia effect of allogeneic T cells. Proc. Natl. Acad. Sci. USA. 87:5633-5637.

19. Dialynas, D.P., et al. 1983. Characterization of murine T cell surface molecule, designated L3T4, identified by monoclonal antibody GK1.5: similarity of L3T4 to human Leu3/T4 molecule. J. Immunol. 131:2445-2451.

20. Sarmiento, M., Glasebrook, A.L., and Fitch, F.W. 1980. IgG or IgM monoclonal antibodies reactive with different determinants on the molecular complex bearing Lyt 2 antigen block $\mathrm{T}$ cell-mediated cytolysis in the absence of complement. J. Immunol. 125:2665-2672.

21. Sykes, M., Abraham, V.S., Harty, M.W., and Pearson, D.A. 1993. IL-2 reduces graft-versus-host disease and preserves a graft-versus-leukemia effect by selectively inhibiting $\mathrm{CD}^{+} \mathrm{T}$ cell activity. J. Immunol. 150:197-205

22. Sykes, M., Romick, M.L., Hoyles, K.A., and Sachs, D.H. 1990. In vivo administration of interleukin 2 plus $\mathrm{T}$ cell-depleted syngeneic marrow prevents graft-versus-host disease mortality and permits alloengraftment. J. Exp. Med. 171:645-658.

23. Unkeless, J.C. 1979. Characterization of a monoclonal antibody directed against mouse macrophage and lymphocyte Fc receptors. J. Exp. Med. 150:580-596.

24. Ozato, K., Mayer, N.M., and Sachs, D.H. 1982. Monoclonal antibodies to mouse major histocompatibility complex antigens. IV. A series of hybridoma clones producing anti- $\mathrm{H}-2 \mathrm{~d}$ antibodies and an examination of expression of $\mathrm{H}-2 \mathrm{~d}$ antigens on the surface of these cells. Transplantation. 34:113-120.

25. Wang, M.-G., Szebeni, J., Pearson, D.A., Szot, G.L., and Sykes, M. 1995. Inhibition of graft-versus-host disease (GVHD) by IL-2 treatment is associated with altered cytokine production by expanded GVH-reactive CD4+ helper cells. Transplantation. 60:481-490.

26. Ohdan, H., Yang, Y.-G., Shimizu, A., Swenson, K.G., and Sykes, M. 1999. Mixed chimerism induced without lethal conditioning prevents $T$ celland anti-Gal $\alpha 1,3$ Gal-mediated graft rejection. J. Clin. Invest. 104:281-290.

27. Krenger, W., Snyder, K.M., Byon, J.C.H., Falzarano, G., and Ferrara, J.L.M. 1995. Polarized type 2 alloreactive CD4+ and CD8+ donor T cells fail to induce experimental acute graft-versus-host disease. J. Immunol. 155:585-593.

28. Heyd, J., Doonenberg, A.D., Burns, W.H., Saral, R., and Santos, G.W. 1988. Immunoglobulin E levels following allogeneic, autologous and syngeneic bone marrow transplantation: an indirect association between hyperproduction and acute GVHD in allogeneic bone marrow transplantation. Blood. 72:442-446. 
29. Claman, H.N., and Spiegelberg, H. 1990. Immunoglobulin dysregulation in murine GVHD: a hyper IgE syndrome. Clin. Immunol. Immunopathol. 56:46-53.

30. Kelso, A. 1995. Th1 and Th2 subsets: paradigms lost? Immunol. Today 16:374-379.

31. Ushiyama, C., et al. 1995. Anti-IL-4 antibody prevents graft-versus-host disease in mice after allogeneic bone marrow transplantation. The IgE allotype is an important marker of graft-versus-host disease. J. Immunol. 154:2687-2696.

32. Sykes, M., Szot, G.L., Nguyen, P.L., and Pearson, D.A. 1995. Interleukin12 inhibits murine graft-versus-host disease. Blood. 86:2429-2438.

33. Brok, H.P.M., Heidt, P.J., Van der Meide, P.H., Zurcher, C., and Vossen, J.M. 1993. Interferon-gamma prevents graft-versus-host disease after allogeneic bone marrow transplantation in mice. J. Immunol. 151:6451-6459.

34. Williamson, E., Garside, P., Bradley, J.A., More, I.A.R., and Mowat, A.M. 1997. Neutralizing IL-12 during induction of murine acute graft-versushost disease polarizes the cytokine profile toward a Th2-type alloimmune response and confers long term protection from disease. $J$. Immunol. 159:1208-1215.

35. Dey, B., Yang, Y.-G., Szot, G.L., Pearson, D.A., and Sykes, M. 1998. IL-12 inhibits GVHD through a Fas-mediated mechanism associated with alterations in donor T cell activation and expansion. Blood. 91:3315-3322.

36. Sykes, M., Abraham, V.S., Harty, M.W., and Pearson, D.A. 1992. Selective inhibition of CD4 graft-versus-host activity in IL-2-treated mice. Transplant. Proc. 25:1225-1226.

37. Sykes, M., and Abraham, V.S. 1992. Mechanism of IL-2-mediated protection against GVHD in mice. II. Protection occurs independently of NK/LAK cells. Transplantation. 53:1063-1070.

38. Sykes, M., Harty, M.W., Szot, G.L., and Pearson, D.A. 1994. Interleukin2 inhibits graft-versus-host disease-promoting activity of CD4+ cells while preserving CD4- and CD8-mediated graft-versus-leukemia effects. Blood. 83:2560-2569.

39. Huang, H., and Paul, W.E. 1998. Impaired interleukin 4 signaling in T helper type 1 cells. J. Exp. Med. 187:1305-1313.

40. Akimoto, T., et al. 1998. Abrogation of bronchial eosinophilic inflammation and airway hyperreactivity in signal transducers and activators of transcription (STAT)6-deficient mice. J. Exp. Med. 187:1537-1542.

41. Kaplan, M.H., Wurster, A.L., and Grusby, M.J. 1998. A signal transducer and activator of transcription (STAT) 4 -independent pathway for the development of T helper type 1 cells. J. Exp. Med. 6:1191-1196.

42. Simpson, J.S., et al. 1998. T cell-mediated pathology in two models of experimental colitis depends on the interleukin 12/signal transducer and activator of transcription (STAT)-4 pathway, but is not conditional on interferon $\gamma$ expression by T cells. J. Exp. Med. 187:1225-1234.

43. Xian, L.C., Zand, M.S., Li, Y., Zheng, X.X., and Strom, T.B. 1998. On histocompatibility barriers, Th1 to Th2 immune deviation, and the nature of the allograft responses. J. Immunol. 161:2241-2247.

44. Takai, S., et al. 1993. Increased IgE level as a marker of host-versus-graft disease: inhibition of this HVGD with a monoclonal antibody to IL-4. Cell. Immunol. 149:1-10.

45. Carter, L.L., and Dutton, R.W. 1995. Relative perforin- and Fas-mediated lysis in $\mathrm{T} 1$ and $\mathrm{T} 2 \mathrm{CD} 8$ effector populations. J. Immunol. 155:1028-1031.

46. Baker, M.B., Altman, N.H., Podack, E.R., and Levy, R.B. 1996. The role of cell-mediated cytotoxicity in acute GVHD after MHC-matched allogeneic bone marrow transplantation. J. Exp. Med. 183:2645-2654.

47. Tsukada, N., Kobata, T., Aizawa, Y., Yagita, H., and Okumura, K. 1999. Graft-versus-leukemia effect and graft-versus-host disease can be differentiated by cytotoxic mechanisms in a murine model of allogeneic bone marrow transplantation. Blood. 93:2738-2747.

48. Miwa, K., et al. 1999. Therapeutic effect of an anti-Fas ligand $\mathrm{mAb}$ on lethal graft-versus-host disease. Int. Immunol. 11:925-931.

49. Sallusto, F., Mackey, C.R., and Lanzavecchia, A. 1997. Selective expression of the eotaxin receptor CCR3 by human T helper 2 cells. Nature. 227:2005-2007.

50. Ward, S.G., Bacon, K.B., and Westwick, J. 1998. Chemokines and T lymphocytes: more than an attraction. Immunity. 9:1-11.

51. Xu, D., et al. 1998. Selective expression of a stable cell surface molecule on type 2 but not type 1 helper T cells. J. Exp. Med. 187:787-794.

52. Bonecchi, R., et al. 1998. Differential expression of chemokine receptors and chemotactic responsiveness of type $1 \mathrm{~T}$ helper cells (Th1s) and Th2s. J. Exp. Med. 187:129-134.

53. Austrup, F., et al. 1997. P- and E-selectin mediate recruitment of Thelper-1 but not T-helper-2 cells into inflamed tissues. Nature. 385:81-83.

54. Lafaille, J.J., et al. 1997. Myelin basic protein-specific T helper 2 (Th2) cells cause experimental autoimmune encephalomyelitis in immunodeficient hosts rather than protect them from the disease. J. Exp. Med. 186:307-312.

55. Pakala, S.V., Kurrer, M.O., and Katz, J.D. 1997. T helper 2 (Th2) T cells induce acute pancreatitis and diabetes in immune-compromised nonobese diabetic (NOD) mice. J. Exp. Med. 186:299-306.

56. O'Garra, A., Steinman, L., and Gijbels, K. 1998. CD4+ T-cell subsets in autoimmunity. Curr. Opin. Immunol. 9:872-883.

57. Chilosi, M., et al. 1996. CD30 cell expression and abnormal soluble CD30 serum accumulation in Omenn's syndrome: evidence for a $\mathrm{T}$ helper 2-mediated condition. Eur. J. Immunol. 26:329-334. 